\title{
Actions de sensibilisation aux nanotechnologies Nano-Ecole IdF
}

\author{
Hugues Cazin d'Honincthun, Sylvain Held, Sylvie Retailleau \\ hugues.cazin@u-psud.fr \\ Adresse : Nano-Ecole IdF, Université Paris-Sud, bat. 220, 91405 Orsay Cedex
}

\begin{abstract}
RESUME : Dans cet article, nous présentons le projet Nano-Ecole Ile-de-France de sensibilisation aux nanosciences et nanotechnologies à destination des écoles, des lycées et du grand public. Nous exposons le format et le détail du contenu des interventions réalisées au sein d'établissements scolaires. Enfin nous présentons la mise en place de formations proposées aux enseignants du secondaire ainsi que les actions à destination des élèves de primaire et du grand public.
\end{abstract}

Mots clés : nanotechnologie, vulgarisation scientifique, travaux pratiques, science et société.

\section{INTRODUCTION}

Dans un contexte de désaffection croissante des jeunes pour les études scientifiques et techniques et principalement pour les longs cursus, la mise en place d'initiatives innovantes pour rendre les sciences plus attractives est un enjeu important. Dans ce cadre, les nanosciences et nanotechnologies, sciences nouvelles touchant de nombreux secteurs d'application et appelées à connaître un fort développement, sont particulièrement adaptées. En effet, suite à la dernière réforme des programmes scolaires, les nanosciences et nanotechnologies sont désormais aux programmes de première et de terminale scientifique spécialité Physique.

Depuis quelques années, un certain nombre de pays, plus particulièrement aux Etats-Unis, se sont intéressés à la manière d'introduire les nanosciences dans l'enseignement secondaire [1]. Les enjeux sont importants et de nature différente car il s'agit comme on l'a dit de former de futur spécialistes du domaine, d'informer les citoyens de demain sur ces nouvelles thématiques dites «sensibles» mais aussi d'utiliser l'interdisciplinarité qui les caractérisent pour motiver et donner une image plus concrète des sciences. Ainsi, aux états Unis, la National Science Foundation (NSF) par le biais du Nanotechnology Center for Learning and Teaching (NCLT) consacre de gros moyens pour introduire les nanosciences dans les programmes, former les enseignants, et de nombreuses études en sciences de l'éducation traitent du sujet en proposant des méthodes pédagogiques pour aborder ce vaste sujet.

Dans ce contexte, des initiatives originales pour rendre la science et la compréhension du monde qui nous entoure plus concrètes existent également en France dans le domaine des nanosciences et nanotechnologies [2] dont l'action Nano-Ecole (Ile-de-France) qui fera l'objet de cet article [3]. Nano-Ecole est un projet national, présent sur 4 pôles (Grenoble, Lille, Paris-Saclay et Toulouse), visant à expérimenter l'introduction des nanotechnologies à l'école en associant enseignement scientifique et réflexion socioscientifique. Ce projet a initialement vu le jour il y a 2 ans dans le cadre du plan en faveur des nanotechnologies Nano-Innov.

\section{PROJET PEDAGOGIQUE}

Le projet pédagogique de Nano-Ecole Ile-deFrance a été construit selon les grands principes définis par le projet Nano-Ecole national tout en exploitant les installations et les compétences des équipes de recherche locales. Dans ce but, l'accent a été mis en premier lieu sur l'interdisciplinarité que nous avons souhaité mettre en avant dans le contenu. Afin d'augmenter l'attractivité du projet, nous avons choisi un format original de présentations théoriques vulgarisées et interactives où les élèves sont invités à participer activement, mêlées à des activités concrètes de mise en application par les élèves qui réalisent eux mêmes les expériences. Dans le cadre de ce projet, les élèves sont également mis en contact avec de gros instruments scientifique de laboratoire et le dialogue avec les chercheurs est favorisé. L'objectif étant à la fois de leur faire découvrir les métiers de la recherche et de changer l'image des chercheurs bien souvent perçus, de leurs propres aveux, comme inaccessibles, austères, incompréhensibles et cantonnés dans leur laboratoire. Enfin, l'accent est mis sur la sensibilisation aux enjeux sociétaux qu'il est essentiel d'aborder dans un sujet soumis à la controverse scientifique telles que le sont les nanotechnologies.

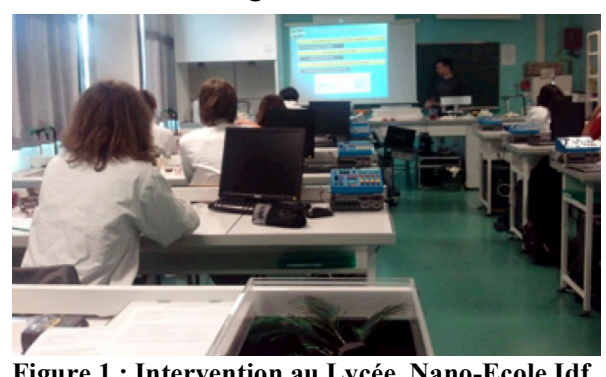

Figure 1 : Intervention au Lycée. Nano-Ecole Idf

Afin de répondre aux attentes des élèves et des enseignants en terme de format et de contenu scientifique, le contenu pédagogique a été développé en collaboration avec des professeurs de Lycée afin de cerner les points d'ancrage entre les grandes idées et concepts fondamentaux des nanosciences et les notions déjà présentes dans le programme scolaire officiel de l'éducation nationale. Cette approche est nécessaire et fortement appréciée des enseignants car cela permet à la fois de revenir sur certaines notions qui ont déjà été 
abordées avec l'enseignant et qu'il est toujours appréciable de revoir, mais également de faciliter l'assimilation des notions nouvelles propres aux nanosciences. De plus, cela permet à nos interventions de mieux s'intégrer dans le programme de l'année et d'en limiter l'impact au regard du nombre d'heures dont les enseignants disposent en lycée et qu'ils jugent bien souvent insuffisantes.

En ce qui concerne le format des interventions, nous avons choisi de répartir l'intervention sur plusieurs séances afin de construire un cheminement dans la transmission des concepts fondamentaux des nanosciences et d'intervenir sur les heures de Travaux Pratiques et donc en demi classes.

\subsection{Mode et déroulement}

Les interventions avec les classes de Lycées se déroulent le plus souvent sur 3 séances de 120 minutes chacune et associent des exposés et des expériences que les jeunes réalisent eux-mêmes. La première séance se déroule au sein de l'établissement scolaire et consiste en une conférence interactive au cours de laquelle les concepts de bases sont exposés, suivie d'une mise en application de ces notions au travers d'un TP de synthèse de nanoparticules d'or réalisée en binôme.

Pour la seconde séance, si l'établissement scolaire le permet, ce sont les élèves qui viennent au sein d'un laboratoire de recherche afin d'y rencontrer des chercheurs, découvrir leur environnement de travail et réaliser quelques expériences.

La dernière séance, qui se déroule à nouveau en établissement scolaire, propose, dans la continuité des séances précédentes, de mettre en évidence des principes fondamentaux des nanosciences à partir d'un TP interactif de microscopie. Il consiste en une initiation à différentes techniques de microscopie, allant du microscope optique jusqu'au microscope à force atomique (AFM), en passant par le microscope électronique à balayage (MEB). Enfin, un exposé-TP sur les couleurs structurelles, traitant notamment des couleurs des papillons morpho bleus, termine cette séance.

Tout au long des 3 séances, les aspects sociétaux sont abordés et pour les établissements intéressés, nous proposons une conférence-débat en collaboration avec les enseignants d'Education Civique Juridique et Sociale (ECJS) et avec Virginie Albe, professeur au Laboratoire STEF de l'ENS de Cachan et membre de la cellule Nano\&Société du Centre de compétence en nanosciences d'Ile-de-France (C'nano IdF).

\subsection{Contenu du projet}

Les thèmes et les contenus matériels proposés ont été développés en collaboration avec le C'nano Ilede-France et des laboratoires de la région. L'originalité du projet repose en partie sur des nano-démonstrateurs portables, sous forme de mallettes, ayant pour objectif de permettre aux jeunes et au grand public de se familiariser avec les nanosciences et leurs applications et d'en illustrer quelques principes fondamentaux.
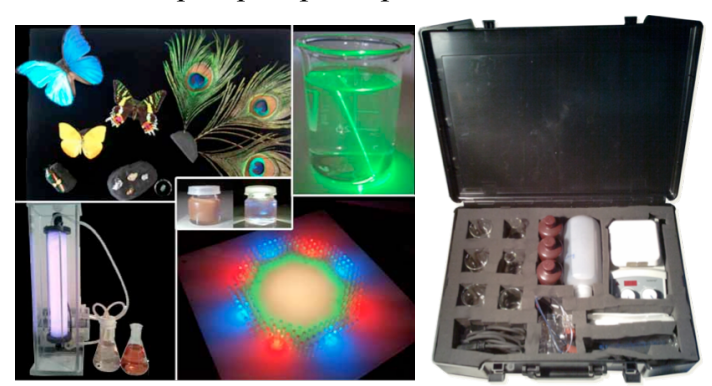

Figure 2 : Démonstrateurs pédagogiques Nano-Ecole Ile-deFrance

Le contenu matériel et pédagogique de ces mallettes est adaptable suivant les conditions d'utilisations et le public visé. Ainsi, l'objectif est de permettre à tout scientifique désireux de faire de la diffusion scientifique de s'approprier rapidement le contenu de la démonstration pour des actions dans le cadre de manifestations scientifiques ou en milieu scolaire. La valorisation de ces mallettes avec la société Jeulin, éditeur de solutions pédagogiques pour l'enseignement scientifique et technique est en cours. On présentera dans cette partie les différents modules de cours qui sont proposés en lycées et qui reposent sur les démonstrateurs développés.

\subsubsection{Initiation aux nanosciences}

En guise d'introduction au Nanomonde, le premier module propose de traiter de généralités sur les nanosciences et les nanotechnologies et d'en exposer les bases.

Il commence par aborder la notion d'échelle et d'ordre de grandeur. Un certain nombre d'études, montrent que les jeunes rencontrent des difficultés importantes à appréhender les petites échelles $[1,4]$. C'est pourquoi nous avons choisi d'aborder la notion d'échelle avec les élèves, sous la forme d'un quizz où ils doivent classer des objets sur une échelle. Il est réalisé à partir de boitiers de vote de type auto-école, ce qui a l'avantage d'être à la fois ludique et de rendre l'apprentissage plus concret.

Quelques concepts de base des nanosciences, aussi connus sous le nom de «Big Ideas » [1] sont ensuite abordés sous la forme d'un exposé interactif agrémenté de séquences vidéo judicieusement choisies. Ainsi, des concepts tels que le changement de comportement et de propriétés de la matière, les nouveaux phénomènes physiques, les notions de rapport surface volume qui ouvrent tout un champ de nouvelles applications possibles dans le domaine des nanotechnologies sont exposés.

L'accent est notamment porté sur une sorte de comparatif entre des applications qui devraient permettre une amélioration de la qualité de vie dans les prochaines années et des applications qui semblent 
moins utiles voire qui pourraient être potentiellement à risque si leur impact sur l'environnement où la santé, aujourd'hui à l'étude, est un jour démontré. Cette réflexion a pour but d'illustrer la notion de rapport bénéfice-risque qui est généralement utilisée dans le domaine de l'évaluation des risques industriels et qui est ensuite discutée avec les élèves.

\subsubsection{Changement de propriétés : exemple des nanoparticules d'or.}

L'objectif de ce module est de mettre en évidence le changement de propriétés de la matière aux faibles dimensions par l'étude des propriétés optiques d'un matériau fascinant et connu de tous: l'or. L'objectif est de partir des connaissances des élèves sur ce matériau et sur ses propriétés de base pour les amener à constater par eux-mêmes que les propriétés optiques sont modifiées en réduisant la taille du matériau dans les 3 dimensions. A partir du matériel de chimie de Lycée usuel (plaque chauffante, béchers, erlenmeyers, pipettes graduées) et d'une fiche de TP, ils réalisent en binôme une synthèse de nanoparticules d'or monodisperses par voie aqueuse, formalisée en 1951 par J. Turkevich [5]. Le principe de cette réaction simple consiste en une réduction de sels d'or (acide tétrachloroaurique de formule $\mathrm{HAuCL}_{4}$ ) par du citrate de sodium en excès $\left(\mathrm{Na}_{3} \mathrm{C}_{6} \mathrm{H}_{5} \mathrm{O}_{7}\right)$. L'avantage de cette expérience est que les réactifs utilisés sont fortement dilués et ne présentent donc pas de risques majeurs pour la sécurité, tant que les protections adéquates sont utilisées et que les règles habituelles de TP de chimie sont respectées.

Suite à une étape de dilution, les élèves obtiennent en quelques minutes une solution colloïdale constituée de nanoparticules d'or de couleur rouge rubis. Le taux d'échec est assez faible à partir du moment où la propreté de la verrerie et la qualité de l'eau distillée utilisée sont respectées. A l'issue du TP, la récupération des déchets nanoparticulaires est bien entendu appliquée et le rinçage dans l'évier interdit. A cette occasion il est souvent bienvenu de discuter des aspects sociétaux et des enjeux environnementaux associés. Le phénomène à l'origine de cette couleur correspond à un effet de résonnance de plasmons de surface localisés dans chaque nanoparticule d'or. L'explication simplifiée du phénomène consiste à dire qu'une nanoparticule d'or a une fréquence de résonnance de ses électrons de conduction qui est fonction de sa taille et de sa forme. Sous l'action d'un champ électromagnétique, telle que la lumière, les électrons entrent en résonnance pour une plage de longueur d'onde donnée. Une partie du rayonnement de la lumière visible est absorbée, et la couleur complémentaire est observée. Des nanoparticules d'or en bâtonnets (nanorods), synthétisées au Laboratoire de Chimie Physique d'Orsay, illustrent que la couleur de ces nano-objets est fortement dépendante de la géométrie de la particule et plus précisément de sa forme et de sa taille étant donnée qu'à chaque dimension de bâtonnet correspond une couleur (voir Figure 3).

Tout au long de ce TP, diverses notions du programme sont abordées telles que la dilution, la conservation de la quantité de matière, l'oxydo-réduction. Des calculs simples sont proposés et permettent d'aborder une réflexion sur le coût et la facilité de fabrication de telles particules et sur la balance bénéfice/risque de leurs applications.

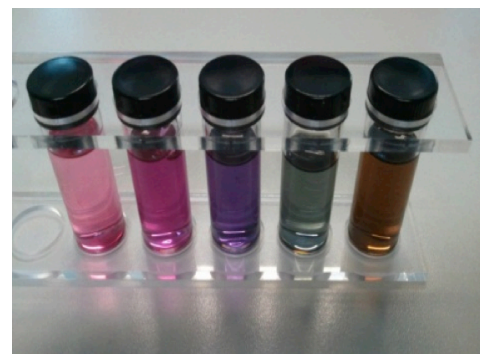

Figure 3 : Solutions de nanoparticules d'or de forme oblongue dont la couleur dépend de la taille (LCP)

Dans un deuxième temps, des exemples concrets d'applications des nanoparticules d'or sont proposés. Une explication complète du fonctionnement du test de grossesse sert de première illustration et des applications plus générales dans les domaines de la biologie et du biomédicale sont ensuite abordées (capteur biologique, test rapide, etc.). Les applications des nanoparticules d'or pour la localisation de tumeurs et le traitement du cancer par hyperthermie sont également présentées en utilisant des notions simples de SVT et ainsi mettre en avant l'interdisciplinarité. Enfin, des exemples de l'utilisation très rependue de nanoparticules métalliques dans le domaine de l'art sont exposés en guise de conclusion.

\subsubsection{La conception de dispositifs}

Ce module, ouvert principalement aux lycéens, se déroule en laboratoire et propose de faire découvrir l'environnement de travail d'un chercheur en nanosciences et les différents métiers d'un laboratoire. Ce type d'action est essentiel à la promotion des sciences, pour changer l'image souvent biaisée qu'ont les jeunes du monde de la recherche, faire découvrir le quotidien des chercheurs et susciter des vocations dans l'espoir de construire la recherche de demain.

La durée étant limitée à $3 \mathrm{~h} 00$ au total, l'objectif est de donner une vision synthétique des techniques et des problématiques de conception de nano-dispositifs. A leur arrivée, les élèves sont pris en charge dans une salle où une présentation leur est proposée. Cet exposé introductif a pour mission de fournir des bases en miniaturisation et de présenter les approches de conception, les étapes technologiques de base et l'environnement de travail spécifique qu'est la salle blanche. Ils sont ensuite répartis en groupe et conduits en salle blanche.

En un temps limité à $2 \mathrm{~h} 00$, les élèves réalisent quelques étapes de fabrications standard (dépôt, litho- 
graphie, gravure) et de caractérisation au sein de la centrale de Technologie Universitaire Minerve de l'Institut d'Electronique Fondamentale d'Orsay. A l'issue de la séance, les élèves repartent avec un échantillon de ce qu'ils ont réalisé.

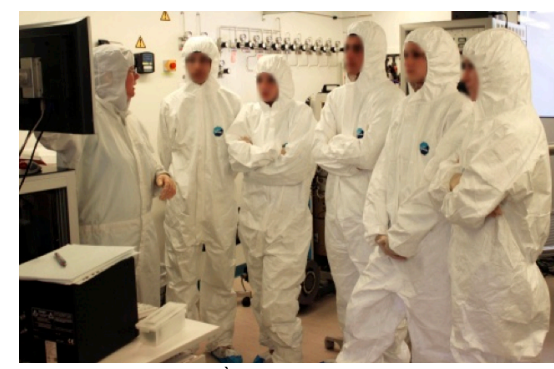

Figure 4 : Des élèves de $1^{\text {ère }} \mathrm{S}$ dans la centrale de Technologie IEF Minerve

Une réorganisation complète de ce module est en cours afin qu'il s'intègre davantage dans la continuité de la première séance avec un travail préparatoire et des objectifs concrets et ciblés étant donné qu'il est difficile en peu de temps d'avoir une bonne vision des techniques de micro et nano-fabrication.

\subsubsection{La microscopie}

Il est bien souvent admis que les nanosciences ont véritablement pu émerger à partir du moment où le microscope à effet tunnel a été inventé et a donc permis de « voir» et « manipuler» les atomes. Dans le cadre d'une action de vulgarisation des nanosciences, où l'on parle de changement de propriétés, de manipulation de la matière et de nanostructures, proposer un module de microscopie afin de «montrer» le nano-monde est essentiel.

L'idée de ce module est de proposer une descente progressive vers l'infiniment petit par une présentation synthétique et interactive qui débute en présentant les différentes techniques existantes et les limitations physiques de chacune. Pour cela, on propose de partir de ce qui est connu de tous, l'œil, pour passer à l'étude du microscope optique après avoir défini des notions telles que le pouvoir de résolution et la limite de diffraction. Partant du constat que les établissements scolaires sont inégalement équipés dans ce domaine, nous avons fait le choix d'utiliser des microscopes en réflexion USB Dino-lite qui sont dotés de grossissements 20-70 et 500 et d'accessoires divers dont des polariseurs. Ils ont l'avantage d'être extrêmement simples d'utilisation, robustes et de permettre l'observation d'objets de diverse nature. En ce qui concerne le choix des échantillons, par soucis de cohérence avec la séance qui suit, qui traite des couleurs structurelles, des ailes de papillons sont observées. Elles présentent une structure en écaille de 50 microns par 100 microns.

Suite à ces observations, l'accent est mis sur la nécessité de passer à une autre technique de microscopie pour descendre un peu plus loin au sein de la matière. Afin de pouvoir faire manipuler les élèves, nous avons fait l'acquisition un microscope électronique à balayage portable TM3000 de marque Hitachi. Cet appareil transportable aux dimensions réduites et au poids acceptable de $60 \mathrm{~kg}$ peut être utilisé en salle de classe et a été financé grâce au soutien de Nano-Innov, du C'nano-Idf et du CNFM. Il est capable d'atteindre une résolution permettant d'observer le nanomonde avec des motifs de l'ordre de la centaine de nanomètre sans trop de difficultés moyennant une préparation adéquate de l'échantillon. Les élèves en quart de groupes, sont invités à utiliser eux mêmes le microscope et à réaliser un ensemble d'observations et de mesures sur les structures des ailes de papillons observées.

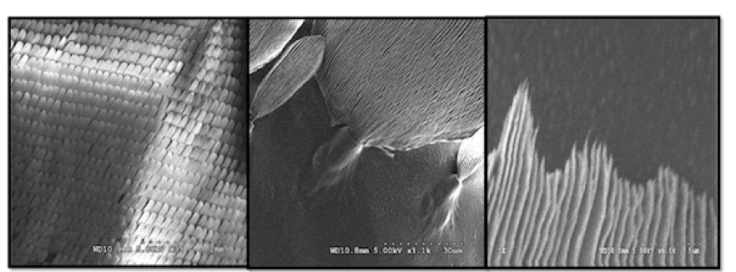

Figure 5 : Observation au microscope électronique à balayage d'ailes de papillons morphos bleus

Afin de n'avoir à gérer que des groupes réduits, des observations en microscopie champ proche sont réalisées en parallèle. L'accent est mis sur cette technique d'imagerie, la plus adaptée et la plus utilisée dans le domaine des nanotechnologies depuis l'invention du microscope à effet tunnel en 1981. La possibilité de structurer la matière avec ce type d'appareil est également mise en avant. Nous avons fait le choix de proposer aux élèves de travailler avec un microscope à force atomique et non pas à effet tunnel. Ce choix s'est fait après avoir constaté que la mise en œuvre du STM avec les élèves était aléatoire, que cela engendrait de grandes difficultés de compréhension des images observées et que les observations bruitées de surface atomique, réalisées dans un environnement peu adapté, avait un impact négatif sur la perception des élèves de cette technologie. Parmi les échantillons utilisés avec l'AFM, on propose le DVD pour lequel les élèves sont invités à observer et mesurer la largeur des pistes. Ces observations et mesures sont ensuite reprises dans le module sur les couleurs structurelles et constituent un objectif à l'atelier au cours duquel les élèves doivent de ce fait être actifs. Cela permet également de mettre en évidence l'intérêt du microscope en tant qu'outil essentiel de recherche permettant de comprendre des phénomènes et de mesurer des objets de l'infiniment petit.

\subsubsection{Les couleurs structurelles}

La plupart des couleurs que nous observons dans la nature sont dites pigmentaires : elles sont liées à la présence de pigments colorés dans l'objet qui absorbent certaines longueurs d'ondes du visible, donc certaines couleurs. Mais, dans le cas des plumes de certains oiseaux, des ailes de papillon et des opales, les couleurs sont structurelles, dites physiques, et donc 
liées à la structure nano/microscopique de l'objet. Ce module s'intéresse particulièrement au phénomène de changement de couleurs qui les caractérisent suivant la manière dont on regarde l'objet, que l'on appelle : l'iridescence [6]. Ces couleurs, largement présentes dans la nature avec pour exemples la plume de paon, l'opale, les papillons morpho bleus ou un objet artificiel tel que le cd, sont directement liées à l'organisation de la matière. Ce sujet s'intègre particulièrement bien dans le programme scolaire étant donné qu'il fait appel à des notions abordées en première scientifique telles que le spectre électromagnétique, l'optique, la dispersion et des notions de terminale telles que les propriétés des ondes, les phénomènes de diffraction et d'interférences.

La compréhension de ce phénomène nécessite en premier lieu de constater que la structure de ces

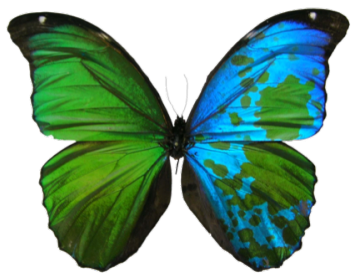

Figure 6 : Expériences sur les couleurs structurelles des papillons morpho bleus objets est périodique et de petite taille, constat qui fait suite à l'étude microscopique réalisée dans le cadre du module correspondant et décrit précédemment. Le second élément abordé concerne le comportement ondulatoire de la lumière rencontrant ces structures périodiques et plus précisément la notion d'interférence et de diffraction. L'explication simplifiée de ces phénomènes combinée à des mises en applications pratiques autour de mesures de diffraction effectuées sur un DVD ou sur un réseau suffisent à faire comprendre comment se forment ce type de couleurs. Des maquettes et expériences inspirées par des travaux de chercheurs de l'Institut des NanoSciences de Paris (INSP) finissent de convaincre de la différence entre couleur pigmentaire et structurelle et donc de la relation entre la structuration et la couleur observée.

A l'issu du module, nous étudions comment on utilise aujourd'hui dans les laboratoires dans le domaine de la nanophotonique, la possibilité de contrôler la lumière grâce à ce type de nanostructures.

\subsubsection{Bilan}

A l'issue de l'intervention, une fiche d'évaluation anonyme est distribuée afin de connaître les aspects à améliorer. Au fur et à mesure des interventions, les retours nous ont permis d'améliorer grandement la cohérence des séances ainsi que leur contenu, afin de permettre que les notions à transmettre soient assimilées par le plus grand nombre. La gestion du temps reste un paramètre difficile à maitriser selon les contraintes de l'établissement (matériel, configuration des salles, etc.) et les différences de niveaux entre les élèves, plus particulièrement en ce qui concerne leur habileté à réaliser les expériences.

Actuellement, l'intérêt et la compréhension des élèves pour ces interventions évoluent aux alentours de $80 \%$.

\section{ACTIONS DE FORMATION}

En plus des actions de formation réalisées à destination des élèves de première et de Terminale, le projet Nano-Ecole intervient auprès des écoles primaires et propose des formations destinées aux enseignants du second degré.

\subsection{En école primaire}

En considérant que les élèves doivent être initiés dès leur plus jeune âge à la curiosité scientifique si on souhaite qu'ils acquièrent le gout des sciences, des interventions en école primaire, sont également dispensées sur le thème de la couleur sous un format original de type "Théâtre et Science » et réparties sur 2 séances. Ce format plus ludique a l'avantage d'être adaptable à tout type d'établissement et permet en particulier d'intervenir dans des classes en milieu défavorisé. Les ateliers se déroulent majoritairement avec classes de CE2, CM1 et CM2.

\subsubsection{Déroulement}

La première séance propose une découverte des couleurs pigmentaires, de la synthèse des couleurs et des notions d'absorption. Tels de vrais petits scientifiques, les enfants vont suivre une démarche expérimentale pour comprendre comment se forment les couleurs et répondre à des questions telles que «Comment et pourquoi un citron est-il jaune?».

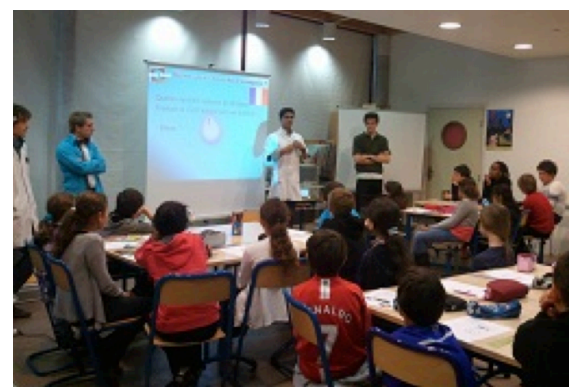

Figure 7 : Initiation aux «couleurs du nanomonde » en école primaire

Cette initiation se déroule au travers d'expérience utilisant des spectrophotomètres à fibre, des kits de synthèse de couleurs utilisant des filtres colorés et autour d'ateliers manuels au cours desquels ils fabriquent des spectromètres en carton à ramener chez eux.

La seconde séance traite des « couleurs du nanomonde » et poursuit dans cette approche d'investigation, avec pour objectif de découvrir que d'autres types de couleurs existent et de déterminer comment se forment ces couleurs. En se limitant à une approche « avec les mains » des phénomènes grâce à du matériel adapté, les élèves sont initiés aux couleurs des papillons et des nanoparticules d'or, assistés par des étudiants de masters. 


\subsubsection{Retours}

Les enfants des cet âge étant en général très enthousiastes vis à vis de ce type d'interventions extérieures, les retours des enfants et des professeurs des écoles sont très positifs et de nouvelles séances sont déjà programmées pour cette année.

\subsection{Formation d'enseignants}

Afin de proposer un accompagnement suite à la récente mise en place de la thématique des nanotechnologies dans le programme des lycées, nous avons proposé cette année une formation de 2 jours dans le cadre du Plan Académique de Formation (PAF) de l'Académie de Versailles sur le thème : «Recherches et avancées en nanotechnologies ».

\subsubsection{Déroulement}

Le but de cette formation est de donner aux enseignants une vision non exhaustive des avancées et découvertes de la recherche dans différents domaines des nanotechnologies telles que la Nanoélectronique, la Nanophotonique, la Nanochimie, les Nanobiosciences et les applications qui y sont associées mais aussi sur les enjeux sociétaux qui sont intrinsèquement liés aux nanotechnologies. La formation est ainsi partagée entre conférences et ateliers pratiques tels que la fabrication de nano-dispositifs en salle blanche et la caractérisation à l'aide de microscopes en champs proche. Des pistes pour aborder ces thématiques en classe leur sont également proposées au travers de démonstrations réalisées à partir de nos démonstrateurs.

\subsubsection{Retours}

$\mathrm{Au}$ mois de mars 2012 avait lieu la première édition de cette formation à laquelle 25 enseignants de l'académie de Versailles se sont inscrits.

Sur 1'ensemble des participants, $75 \%$ des présents étaient enseignants en Lycée, le reste en collège. Ils étaient en très grande majorité enseignants de Physique-Chimie dans les 3 classes : $2^{\text {nde }}, 1^{\text {ere }}$ et Terminale dont $25 \%$ en spécialité Physique. Leurs attentes sont nombreuses et diverses, mais ils recherchent en grande majorité à connaître l'état de la recherche dans le domaine des nanotechnologies et du contenu directement exploitable dans le cadre de leurs enseignements.

$\mathrm{Au}$ niveau des perspectives, $100 \%$ des participants proposent de renouveler ce stage et donc de le généraliser. A noter que $73 \%$ pensent donner une suite à ce stage dans leur établissement ce qui est également très encourageant.

Fort de son succès et des bonnes évaluations des enseignants, le rectorat a reconduit la formation pour 2012-2013.

\section{ACTIONS ENVERS LE PUBLIC}

De nombreuses actions envers le grand public ont ponctué cette année. Les kits-démonstrateurs NanoEcole IdF sont particulièrement adaptés à ce genre de manifestations où l'initiation à la microscopie, les animations autour des couleurs structurelles et les expériences sur différents types de nanoparticules émerveillent petits et grands et favorisent de nombreux échanges sur les aspects sociétaux. Un réacteur compact de dépollution de l'eau au $\mathrm{TiO}_{2}$, développé par des chercheurs du Laboratoire de Chimie Physique de l'Université Paris-Sud est tout particulièrement adapté pour engager le dialogue sur les enjeux environnementaux des nanotechnologies et sur la présence des nanoparticules dans notre quotidien aujourd'hui et demain. Nous avons participé cette année à une dizaine de manifestations scientifiques (Fête de la science, Fête de la quantique au musée du CNAM, Nuit des chercheurs, salons, forums et expositions) à l'occasion desquelles plusieurs milliers de personnes étaient présentes.

\section{CONCLUSION}

Cette année, les actions Nano-Ecole IdF ont été menées auprès de 25 enseignants dans le cadre du Plan académique de formation PAF, 160 élèves de primaire sur 3 écoles et plusieurs centaines de lycéens, des milliers de personnes rencontrées lors de manifestations scientifiques grand public. Alors que nous entamons la troisième année de ce projet ambitieux, et que le contenu du projet semble avoir acquis une certaine maturité et une certaine reconnaissance, l'objectif pour cette année 2012-2013 est d'en poursuivre le développement et d'en élargir le champ d'action à davantage d'établissements de la région Ile-de-France. Cela ne sera possible qu'en développant notre réseau avec les différents partenaires régionaux et nationaux impliqués dans ce type d'actions.

\section{Bibliographie}

[1] B. Hingant, V. Albe, "Nanosciences and nanotechnologies learning and teaching in secondary education : ar eview of literature", Studies in Science Education, Vol. 46, 2 (2010), DOI 10.1080/03057267.201.504543.

[2] N. Panissal, J.C. Cau et al., "Nanotechnology training before university: a new approach combining scientific and social issues", Journal of Materials Education, Vol. 33, 1-2 (2011).

[3] www.nano-ecole.fr

[4] C. Delgado, S. Stevens, et al., "The development of students' conceptions of size", Paper presented at the annual meeting of the NARST (2007).

[5] J. Turkevich, P. C. Stevenson, J. Hillier, "A study of the nucleation and growth processes in the synthesis of colloidal gold", Discuss. Faraday. Soc., 11, 55-75 (1951).

[6] S. Berthier, E. Charron, J. Boulenguez, "Morphological structure and optical properties of the wings of Morphidae", Insect Science, Volume 13, Issue 2, pages 145-158, (2006). 